\title{
Research on Ontology Model of the Urban Critical Infrastructure System Interdependence
}

\author{
Shiying Wang, 2 \\ ${ }^{1}$ Department of Management \\ Harbin Institute of Technology \\ P.R.China \\ ${ }^{2}$ Computer Science and Information Engineering \\ Heihe University \\ P.R.China
}

\author{
Xiangyang Li* \\ Department of Management \\ Harbin Institute of Technology \\ P.R.China
}

\author{
Guanghua $\mathrm{Yu}$ \\ Computer Science and Information Engineering \\ Heihe University \\ P.R.China
}

\begin{abstract}
The paper uses ontology method to describe complex interdependency in urban Critical Infrastructure System (CIS). Through the literature review, existing research results have not been excavated the essential causes and influencing factors of the urban CIS interdependency formation. Because the complex network interdependencies in the urban CIS has deep connotation, and the complexity increases with the urban CIS scale. In the research of urban CIS interdependency, attributes such as interdependent nodes and interdependent edges should be taken seriously also, and the data and information contained in them should be refined. The ontology theory can meet above requirements. The paper establishes an interdependency ontology model to generally express the urban CIS interdependency.
\end{abstract}

Keywords—ontology; CIS; interdependency; top-level ontology; application ontology

\section{INTRODUCTION}

The urban is a large settlement which forms by nonagricultural industries and non-agricultural population. The area has dense population and development industrial, and it is usually the center of politics, economy and culture in the surrounding area. Critical Infrastructure System (CIS) is the basic material foundation of urban existence and the cornerstone of modern society. Critical infrastructure refers to the failure or destruction of the systems and assets which exist by physical way or abstraction will seriously affect people's livelihood, economic development, social stability and national security ${ }^{[1]}$. With the continuous development of engineering technology and information technology, critical infrastructure system becomes complex and large, and some critical infrastructures are frequently related ${ }^{[2]}$, such as power networks and water networks, power networks and gas transmission networks, water networks and gas transmission networks. The failure of these critical infrastructures always appears in the same emergency. The reason is that the failure of a critical infrastructure in a critical infrastructure system produces a serious cascade reaction to the other critical infrastructures which are physically overlapping or adjacent to space $^{[3]}$. Ontology can use to describe complex interdependency in urban Critical Infrastructure System.

Ontology which originated from the philosophical category and began in the 17th century by the German philosopher is an objective description of the real existence in any field of the world ${ }^{[4]}$. There are many definitions of ontology. Gruber pointed out in 1993 that ontology is an explicit specification or representation of conceptualization ${ }^{[5]}$. The definition given by Bost in 1997 is that "ontology can be defined as a formal specification of the shared conceptualization". Domestic scholars Liu Ruling etc. given a more practical definition of ontology: "ontology is formal and illustrative expressions about a topic, including its domain of discourse, the object name of domain of discourse, definition and relationship to each other" ${ }^{[6]}$. With the development of ontology, ontology modeling meta-language for different knowledge modeling has been continuously developed and applied in the field of emergency management to indicate disaster events and support for emergency decision-making. Baishang Zhang et al. proposed a four-dimensional ABC model including Abstraction, Place, Actuality and Temporality, and constructed the emergency case ontology model by combining case reasoning features ${ }^{[7]}$. The ontology modeling meta-language proposed by Perez is composed of concepts, relationships, functions, axioms and instances, and it can design and implement the construction and reuse of the emergency case ontology model ${ }^{[8]}$. In addition, scholars used ontology to model the urban CIS interdependency ${ }^{[9]}$.

Supported by the National Natural Science Foundation of China (NSFC) under the Grant Nos.91024028，91024031，91324018and the Social Science and Humanity on Young Fund of the Ministry of Education under the Grant No. 18YJCZH175. 
Based on previous studies, this paper determines the key elements of urban CIS interdependency, and designs a set of urban CIS interdependency ontology modeling meta-language according to the key elements and the disaster theory. At last, design the top-level ontology and the application ontology of urban CIS interdependency and apply to the real disaster.

\section{KEY ELEMENTS OF URBAN CIS INTERDEPENDENCE}

Urban CIS interdependence elements set $D O$ is defined as $D O=\left\{T, V_{p}^{(i)}, V_{q}^{(j)}, A^{(V)}, R, A^{(R)}, H, X\right\}$, which is made up of 8 elements. Among them, the interdependence elements set $D O$ describes the interdependence between $V_{p}^{(i)}$ and other nodes; $T$ is timestamp to represent the time window property of the interdependence ontology; $V_{p}^{(i)}$ is the node of subsystem in urban CIS; $V_{q}^{(j)}$ is the interdependence node with $V_{p}^{(i)}$ in urban CIS; $A^{(V)}$ shows the attribute set of each node; $R$ represents the interdependence style between interdependence nodes; $A^{(R)}$ is interdependence style; $H$ means interdependence level of interdependence nodes; $X$ indicates the interdependence rules of interdependence nodes.

\section{The Ontology Model of Urban CIS INTERDEPENDENCE}

Urban CIS interdependence contains 8 major elements and using ontology method to establish a unified and generic representation model which store in computer by standardized way to realize information integration and sharing within the territory to solve general problems such as information inconsistency and information island. It is facilitation to the development of research work. There are many kinds of ontology description language. OWL has the most complete information and the highest standard through analysis, so selecting the OWL language to describe which is consisted of six major elements named class, data type, object attribute, data attribute, individual and value. Ontology modeling tool Protégé provides many knowledge model architectures which can be used to manipulate various forms of ontology. Therefore, it can be readily accepted by computer to read by using Protégé to construct urban CIS interdependence ontology to show the sharing of knowledge in the field of urban CIS protection.

\section{A. Ontology Modeling Meta-language}

In the field of emergency management, the meta-language always adopts five-dimensional modeling meta-language which is inductive by Perez et al, that is Concept, Relation, Function, Axiom and Instance. However, for urban CIS interdependence modeling, the concept can be further divided into classes. The class performance is different in different states, so it needs attributes and values to describe. The data styles of class are diverse, and the value is constantly changing which the differences in the range may also affect the final vulnerability assessment, so this model needs to introduce three new elements. Based on the above models which are respectively Class, Attribute and Value to construct urban CIS interdependence ontology (UCISOntology). Through classing concepts, establishing subclass, and combining with certain interdependence, it forms the urban CIS interdependence ontology as follow:

$$
\text { UCISOntology }=<\text { Concepts, Class, Relations, Functions, }
$$

Attribute, Value, Axioms, Instances >

- Concept: Urban CIS interdependence ontology core concepts are divided into three aspects which are critical infrastructure described, interdependence description and source of vulnerability description from the view of the interdependence description of the urban CIS, denote as

- $\quad$ UCIS _ Concepts $=\left\{C_{1}, C_{2}, \ldots, C_{i}, \ldots, C_{n}\right\}$;

- Class: Represents the set of entity objects under the concept, that is, the composition of concepts. For example, the concept of critical infrastructure can indicate the type of critical infrastructure as power network class, water network class; the concept of interdependence can be divided into geographical interdependence class, physical interdependence class and information interdependence class; The source of vulnerability can be divided into component disaster class and structural disaster class according to its inherent vulnerability and can be divided into natural disaster class, people disaster class and technical disaster class according to the external environment. The different the focus chosen, the different the classification. The special form is UCIS_Class $=\left\{\left(c_{l}, c l l, \ldots, c l, \ldots, c l l\right) \rightarrow c i \mid c i \in U C I S \_\right.$Concepts $\}$ and classes can also be further subclassed.

- Attribute: The set describes the features of concepts and classes to distinguish different concepts and classes. For instance, geographical interdependence is often associated with the geographic location of critical infrastructures and the distance between them. The set remarks that: UCIS_Attribute $=\left\{\left(a_{1}, a_{2}, \ldots, a_{i}, \ldots, a_{n}\right) \rightarrow\right.$

$c_{i}$ or $C l_{i} \mid c l_{i} \in U C I S$ _Class, $c_{i} \in U C I S$ _Concepts $\}$.

- Value: The specific value of the attribute in the individual instance. The different the attributes, the different the data types. They are can be precise, interval, logical, symbol, or textual, remember to: UCIS_Value $=\left\{\left(v_{1}, v_{2}, \ldots, v_{1}, \ldots, v_{n}\right) \rightarrow a_{i} \mid a_{i} \in U C I S \_\right.$Attribute $\}$.

- Relation: Represents the interdependence or interaction between elements of interdependence ontology which has a wide range of semantics. For example, is-part-of, is-attribute-of, is-instance-of, atTime, inPlace, use, implement, follow, hasImpact, disposal, hasUtility and hasResult. Take the relationship between concepts as an example which can 
be remarked to:

UCIS _ Relations $=\left\{R\left(c_{1}, C_{2}, \ldots, c_{i}, \ldots, C_{n}\right) \mid c_{i} \in U C I S_{-}\right.$Concepts $\}$ In order to reduce the complexity of the research, the multivariate interdependence is usually transformed into a binary interdependence such as

UCIS _ Relations $=\left\{R\left(c_{1}, C_{2}\right) \mid C_{1}, C_{2} \in\right.$ UCIS _ Concepts $\}$.

- Function: Describe concepts and their characteristic attributes, attribute values and the expression of interaction between interdependences. It also indicates that several factors jointly play a decisive role in the qualitative change of a certain factor, such as if the distance of two critical infrastructures is within the range of geographical interdependence function, it may cause geographical interdependence, that is:

UCIS _ Functions $=\left\{f:\left(c_{1}, c_{2}, \ldots, C_{i}, T\right) \rightarrow C \mid C_{i}, T \in U C I S\right.$ _Concepts\}

- Axiom: Said in any case, as long as meet the interdependences and corresponding function rules, the conclusion about the urban CIS interdependence is always true. For example, critical infrastructure $i$ inputs physical resources to critical infrastructure $j$ to ensure the proper functioning of critical infrastructure $j$, we can know that there is a physical interdependence between critical infrastructure $i$ and critical infrastructure $j$, that is

$$
\begin{aligned}
& \text { UCIS _ Axioms }=\left\{A:\left(C_{1}, C_{2}, \ldots, C_{i}\right) \rightarrow C \mid C_{i} \in\right. \text { Concepts, } \\
& C \in U C I S \text { _Concepts } \cup \text { UCIS _ Relations }\} \text {. }
\end{aligned}
$$

- Instance: Instance which is the instantiation of concept content means the individual instances set of various disaster and accident related to urban CIS. For instance, the Dalian oil pipeline explosion in 2013 affected energy network and drainage network at the same time due to geographical interdependence. Depending on the angle of analysis, it can be a technological disaster instance, or a geographical interdependence instance, or an energy network and a drainage network instance, or a regional partition instance. It can be shown as:

UCIS_ Instances $=\left\{c_{i} \rightarrow\right.$ individual $\mid c_{i} \in U C I S_{-}$Concepts $\}$

Though the above meta-language design, the model can comprehensively describe the interdependence of urban CIS and further refine it on the basis of structure and formalization. In the practical application, it is necessary to combine the context of the context to express $t$ concrete form of related urban CIS interdependences according to different disasters.

\section{B. The Top-level Ontology of Urban CIS Interdependence}

Urban CIS interdependence top ontology (UCISOntology_Top) also known as the upper ontology or general ontology is used to represent the objective existence of the urban CIS interdependence which does not depend on any event. UCISOntology_Top could describe the general concept of urban CIS interdependence field, the specific content of the concept and general interdependence to achieve the expansion, integration and interaction from the top-level ontology to the application ontology. At present, the ABC modeling method always is used to develop emergency case ontology model to improve the understanding and utilization of emergency case information and clearly state the concepts and complex interdependences in related fields. The eABC ontology model is proposed based on the extension of the $A B C$ model. It makes the expression more accurate and comprehensive by adding the corresponding concept. Now, establishing urban CIS interdependence top-level ontology which is based on $\mathrm{ABC}$ ontology model and combines interdependence elements contains five concepts as follow:

- Actuality: Describe entity objects that exist in the objective world such as Critical Infrastructure, Physical Resource.

- Temporality: Describe an entity with temporal variability such as Disaster Level, Damage Level, and Vulnerability.

- Abstraction: The concepts which depict by human subjective consciousness such as Interdependency, Experience and Knowledge, Social Effect, and Constraint.

- Situation: The content such as Disaster type, Weather, Geography and Event Chain.

- Event: Refers to the disasters related to the urban CIS and provides the template for the scenario description. For example, Gansu Zhouqu debris flow disasters in 2010 leads to the failure of communication network, power network and transport network one after another and form the spread of mesh by means of interdependence. According to the above natural disasters, the CIS interdependence ontology can be described.

\section{The Application Ontology of Urban CIS Interdependence}

The application ontology of urban CIS interdependence (UCISOntology_Applied) can be developed towards field knowledge based on the basic framework of urban CIS interdependence proposed by the top-level ontology and combining practical instances at the same time. The UCISOntology_Applied is the refinement and expansion of the top-level ontology to the urban CIS interdependence. According to the disaster system scenario theory ${ }^{[10]}$, disaster situation knowledge expression needs to introduce the concepts such as hazard, body, environment, and other concepts. The related knowledge about interdependence still needs to be considered. Therefore, UCISOntology_Applied should be defined as a quaternion group:

\section{UCISOntology _ Applied $=<$ Hazard, Body, Environment,} Interdenpendency > 
Among them, Hazard can be divided into component disasters, structural disasters, natural disasters, human disasters and technical disasters according to the source of vulnerability; Body mainly refers to the urban critical infrastructures; Environment is made up of each state attributes when disasters occurred. Interdependency includes geographical interdependency, physical interdependency, information interdependency and logical interdependency.

\section{CASe AnAlysis}

When constructing of urban CIS interdependency application ontology, the application ontology model is from the extraction of above concepts. The model is composed of three-level concepts. The three-level concepts contain several characteristic attributes, and each attribute has a corresponding specific values name attribute value. The data type of each attribute value is different depending on the attribute. Due to the ontology model will be used to intelligent computer, on the basis of computer data type, the data type can be divided into time date, numeric, character, logic, etc. Taking Dalian oil pipeline explosion at 18:30 on June 30, 2014 as an example to establish urban CIS interdependency application ontology case. According to the information released by China national petroleum corporation in the early hours of July 1, 2014, the main course of the event is as below: Dalian yueyin construction engineering co., LTD., conducts horizontal directional drilling near luan parking lot in jinzhou new district road. It drills into the oil pipeline of petro china and results in oil leakage. Overflow oil flowing into the municipal sewage pipe network leads to burst into flames, the dense petro diffuses in Dalian Jinzhou development zone, and the oil in pipeline returns to the ground from underground water pipeline which is causing a fire. The flame was completely extinguished until 22:20 and no casualties were reported. The corresponding model is shown in Fig. 1.

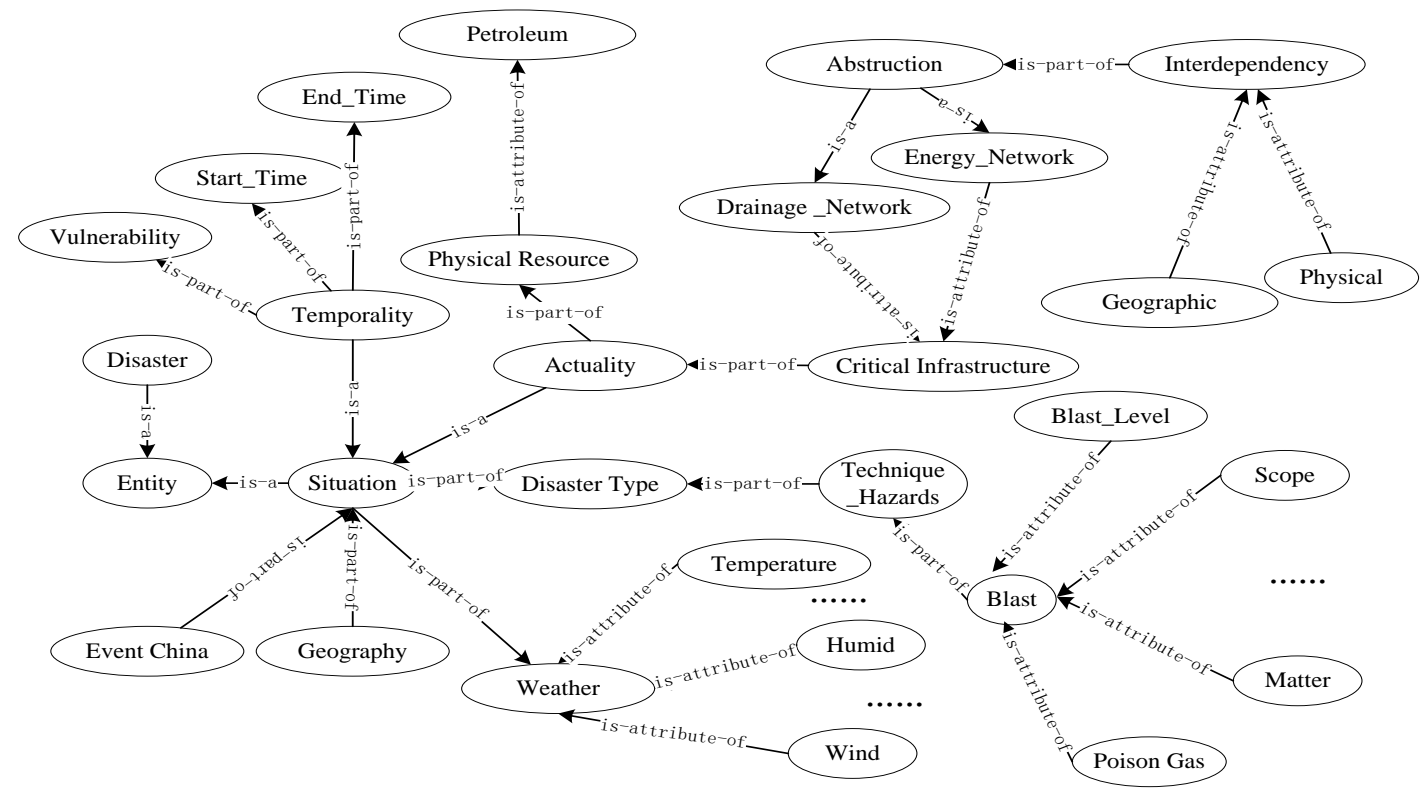

Fig. 1. Example of urban CIS interdependency applied ontology

\section{CONCLUSION}

The paper constructs and designs the urban CIS interdependency ontology. First, define Urban CIS interdependence elements set $D O$ which is made up of 8 elements. They are timestamp, node of subsystem, interdependence node; nodes attribute set, interdependence type, interdependence style, interdependence level, and interdependence rule. Second, based on key elements of urban CIS interdependency, construct two layers ontology modeling and divided into the top-level ontology and the application ontology. The top-level ontology contains five parts such as actuality, temporality, abstraction, situation and event. The application ontology is made up four parts such as hazard, body, environment, and interdependency. Last, use the ontology model of urban CIS interdependence to Dalian oil pipeline explosion disaster.

\section{REFERENCES}

[1] D.A. Jones, M.A. Turnquist, "Simulation of imperfect information in vulnerability modeling for infrastructure facilities," Proceedings of the 2005 Winter Simulation Conference, USA: WSC, pp. 965-971, 2005.

[2] R. Zimmerman, "Social Implications of Infrastructure Network Interactions,” Journal of Urban Technology, 2001, vol.8, pp. 97-119.

[3] S. Jia, S.V. Buldyrev, S. Havlin, "Cascade of Failures in Coupled Network Systems with Multiple Support-dependence Relations," Physical Review E, 2011, vol. 83(3), pp.1-9.

[4] N. Guarino, P. Giaretta, "Ontology an Knowledge Bases-towards a Terminological Clarification,” Towards Very Large Knowledge BasesKnowledge Building and Knowledge Sharing, ISO Press, 1995, pp.2532. 
[5] N. Guarino, "Formal Ontology and In formation System,” Proceedings of FOIS’98, Trento, Italy, 1998, pp. 3-15.

[6] L.U. Ruqian, “Artificial Intelligence” Beijing: science press, 2002, pp. 59-78.

[7] Z. Baishang, L. Xiangyang, L. Jun, "Research on Emergency Case Ontology Model Based on ABC Ontology,” 2013 International Conference on Management Science \& Engineering, Harbin, P.R.China, 2013, pp. 227-233.
[8] K. Gro linger, M.A. Capretz, "Federated Critical Infrastructure Simu lators: Towards Ontologies for Support of Collaboration," Niagara Falls, Canada, 2011, pp.1503-1506.

[9] W. Shuliang, Y. Xin, "Vulnerability Analysis of Interdependent Infrastructure Systems,” Application Research of Computers, 2014, vol.31 (4), pp. 976-981. (In Chinese)

[10] L. Vleuten, “Transnational Infrastructure Vulnerability: The Historical Shaping of the 2006 European Blackout,” Energy Policy, 2010, vol. 38(4), pp. 2042-2052. 